\title{
Eyelid Complications Associated with Surgery for Periocular Cutaneous Malignancies
}

\author{
${ }^{1}$ Department of Ophthalmology, Kellogg Eye Center, University of \\ Michigan, Ann Arbor, Michigan \\ 2 Department of Ophthalmology, Canton Hospital Zenica, Zenica, \\ Bosnia and Herzegovina \\ ${ }^{3}$ Consultants in Ophthalmic and Facial Plastic Surgery, Southfield, \\ Michigan
}

Anaïs L. Carniciu, MD ${ }^{1}$ Nina Jovanovic, $\mathrm{MD}^{2}$ Alon Kahana, MD, $\mathrm{PhD}^{1,3}$

Address for correspondence Alon Kahana, MD, PhD, Consultants in Ophthalmic and Facial Plastic Surgery, 29201 Telegraph Rd. Suite \#324, Southfield, MI 48034 (e-mail: akahana@facialworks.com).

Facial Plast Surg 2020;36:166-175.

\begin{abstract}
Keywords

- eyelid

- basal cell

- squamous cell

- Mohs

- eyelid retraction

Periocular skin is highly prone to malignancies, especially basal cell and squamous cell carcinomas. Because of the complex anatomy and eye-protecting functions of the periocular tissues, treatment of these cancers requires special considerations. Mohs micrographic surgery is usually the treatment of choice, whenever possible, in order to enhance margin control while limiting collateral damage to nearby normal structures. Cancer excision, whether by Mohs or other techniques, will leave a complex defect that requires careful anatomical and functional reconstruction. This study presents some of the challenges of treating periocular skin cancer and associated reconstructive surgery and provides an intellectual framework for addressing these challenges. The key topics are adherence to anatomical landmarks and aesthetic units, proper distribution of tension, and matching the correct reconstructive approach, that is, type of flap or graft, to the defect at hand. This review is not meant to be exhaustive, but it will provide both basic and advanced considerations.
\end{abstract}

Periocular cutaneous malignancies, that is, basal cell carcinoma, squamous cell carcinoma, and, to a lesser extent, melanoma, are common malignancies affecting the eyelids and surrounding tissues. Cure typically requires surgical excision. Such surgery, by necessity, will damage normal eyelid anatomy and function, with cosmetic repercussions as well. Depending on the size of the tumor, excision might result in a tissue defect that requires surgical reconstruction to restore/preserve normal form and function. The surgical techniques employed in the excision and reconstruction may have a significant bearing on the results. Matching the technique to the goals of surgery and performing that technique with skill and attention to detail will usually result in a good outcome. The goal of this study is to review pearls and pitfalls in the surgical care of periocular cutaneous malignancies.

The normal function of the eyelid is to protect the ocular surface without interfering with the visual axis. Excising skin malignancies and performing subsequent reconstruction in the delicate periocular region must consider ocular surface protection, which requires good coverage by the eyelids, hydration, and lubrication. Postsurgical changes that cause eyelid malposition, due to a combination of new cicatricial and preexisting involutional changes, can threaten the health of the ocular surface. ${ }^{1}$

Removal of periocular cutaneous malignancies can be performed by Mohs surgery or other direct surgical techniques. A key advantage of Mohs surgery in the periocular region is the reduction in collateral damage required to achieve a cure. ${ }^{2}$ Irrespective of excisional technique, the eyelid anatomy needs to be restored to preserve form and function. A multidisciplinary approach is sometimes required, particularly in cases involving large facial cutaneous malignancies, to maximize the functionality of individual aesthetic units of the face. ${ }^{3}$ Eyelids are anatomically linked to the forehead/brow (upper eyelid), cheek (lower eyelid and lateral canthus), and nose and lacrimal system (medial canthus). Since the eyelids are also
Issue Theme Management of Melanoma and Advanced Non-Melanoma Skin Cancers of the Face; Guest Editor: Jeffrey S. Moyer, MD
Copyright $\odot 2020$ by Thieme Medical Publishers, Inc., 333 Seventh Avenue, New York, NY 10001, USA. Tel: +1(212) 760-0888.
DOI https://doi.org/ 10.1055/s-0040-1709515. ISSN $0736-6825$. 
critical aesthetic units, any surgery should aim to restore normal aesthetics and avoid deformity ${ }^{4}$ (- Fig. 1). The expertise of an oculofacial plastic surgeon with focused training on
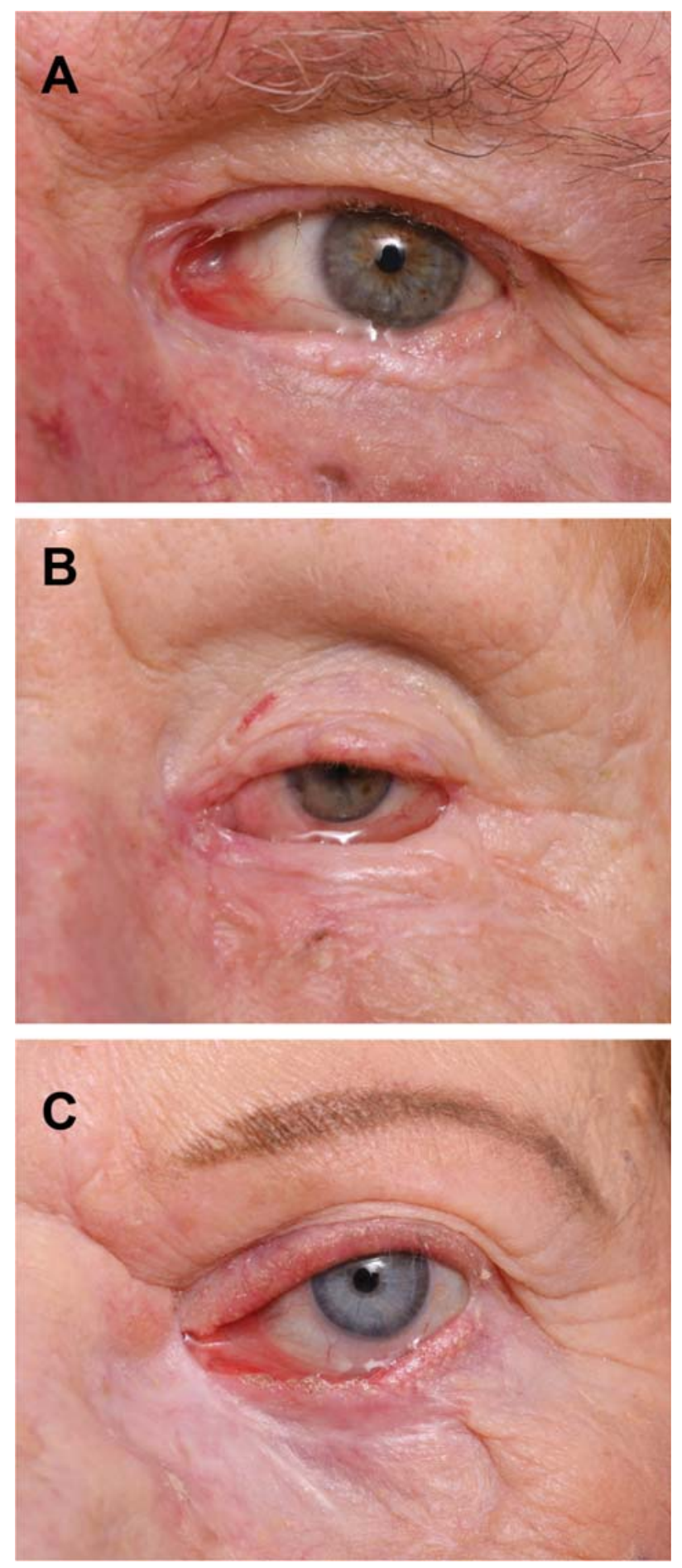

Fig. 1 Medial eyelid retraction and tearing. The most common site for problematic recurrent cutaneous malignancy is the medial canthus. (A-C) These patients all had medial canthal basal cell carcinoma that recurred after an excision by an outside surgeon that did not use Mohs micrographic surgery. In all three cases, the recurrent cancer extended onto the medial aspect of the upper eyelid. Additional surgery required sacrificing the lacrimal drainage apparatus and resulted in acceptable lower eyelid retrac tion. Note the elevated tear lakes in all patients. Medial canthal cutaneous malignancies are particularly challenging, and Mohs micrographic surgery as a first-line treatment is essential for achieving local control. the aesthetic and functional management of eyelid disorders is particularly helpful in these cases.

This article discusses the major lower and upper eyelid complications that may arise following surgery for periocular skin cancer. Additionally, pearls for avoiding complications and managing complications that do arise will be reviewed.

\section{Lower Eyelid Complications}

The lower eyelid is minimally mobile but absolutely essential to the protection of the ocular surface and maintenance of the normal aesthetic unit. The key burden of the lower eyelid is gravity and the close association of the lower eyelid with the cheek through cutaneous contiguity and through the superficial musculoaponeurotic system. ${ }^{5}$

\section{Cicatricial Ectropion}

Cicatricial ectropion (outward turning of the eyelid margin) typically results from the shortening of the anterior lamella (skin and orbicularis muscle) of the lower eyelid. Unlike involutional or age-related causes of ectropion, manual elevation of the lower lid often cannot correct the associated lid malposition due to underlying scarring. Maneuvers that place the lower lid on increased stretch, such as asking the patient to open the mouth or look up, can accentuate cicatricial ectropion. Following lower eyelid tumor excision and repair, the incidence of ectropion was reported to be $14.2 \%$ in a series of 106 consecutive cases over a 7-year period. ${ }^{6}$ However, results are highly dependent on technique and surgeon, and in a report of another series of patients undergoing eyelid reconstruction using tarsoconjunctival grafts, cicatricial ectropion occurred in only 1 of 44 patients. $^{7}$

\section{Cicatricial Entropion}

Conversely, cicatricial entropion can arise in cases of posterior lamella (conjunctiva and tarsus) shortening. This is often caused by conjunctival scarring following excision and repair of lower eyelid defects without addressing the posterior lamellar component. Patients with predisposing conditions such as chronic blepharoconjunctivitis or long-time glaucoma topical therapy users ${ }^{8}$ are at a higher risk of inflammatory changes that result in fibrosis, mucosal scarring, and subsequent lid malposition. Stevens-Johnson's syndrome, ocular cicatricial pemphigoid, and other cicatrizing conjunctival disorders often lead to cicatricial entropion and trichiasis independent of any cancer surgery, which can only worsen a challenging situation. Cicatricial entropion is often associated with other sequelae of chronic inflammation, including keratinization, forniceal shortening, symblephara, and eyelid margin distortion. ${ }^{9}$

\section{Lateral Canthal Disruption}

The lateral canthus can become displaced following excision of cutaneous malignancies and subsequent periocular reconstruction. The normal sharp anatomy of the lateral commissure, where the upper and lower eyelids meet temporally, can become rounded following surgical intervention in the area. Lack of tissue, unrecognized negative vector (malar hypoplasia), 
and overly aggressive skin advancement in the area may additionally cause tractional and cicatricial changes that distort the normal position of the lateral canthus, leading to lateral canthal dystopia. These changes may also cause associated inferior scleral show that distorts the normal oculofacial appearance of reconstructive patients. ${ }^{10}$

\section{Eyelid Retraction}

Lower eyelid retraction is present if the eyelid margin is positioned inferiorly to the inferior aspect of the corneosceral junction (limbus) with the globe in the primary position and the lid margin well apposed to the globe surface. Patients may present with tearing, irritation, and lagophthalmos. Cicatricial postsurgical changes following lower lid cutaneous malignancy excision and repair may result in lower lid retraction, particularly if there is significant vertical tension on the wound. A multifactorial etiology is often the culprit and involves scarring between the orbital septum and lower lid retraction, as well as predisposing lateral canthal tendon laxity, midface descent, and malar hypoplasia. ${ }^{11}$ The role of orbital septum (i.e., "middle lamella") scarring in the pathogenesis of lower eyelid retraction has led to significant improvements in reconstructive techniques, both primary and secondary. ${ }^{12}$

\section{Lagophthalmos}

As suggested earlier, lagophthalmos (incomplete eyelid closure) can present in patients with predisposing eyelid positional changes, typically retraction, following surgery for periocular cutaneous malignancies. Patients may demonstrate concomitant inferior scleral show and are often symptomatic with ocular surface irritation, keratopathy, and reflex tearing. A subset of patients may present with lagophthalmos only while sleeping (nocturnal lagophthalmos). ${ }^{13}$ A normal Bells phenomenon, where the eye rolls upward as the upper eyelid closes, can protect the cornea despite inferior scleral show and lagophthalmos. However, some patients lack a good Bells phenomenon, and the reconstructive surgeon should check for the presence of Bells preoperatively to tailor the surgery to the particular needs of the patient: patients without a good Bells phenomenon will benefit from a more elevated lower eyelid margin post reconstruction.

\section{Notching}

Lower eyelid notching may present in patients with a history of eyelid margin malignancies that have been excised and reconstructed without proper margin eversion in cases of early lost sutures and wound infection. As the tissue margins heal, a notch may develop at the site of repair if excess wound tension is present. ${ }^{14}$ In a study by Hawes and Jamell, notching was one of the more commonly encountered complications of eyelid reconstruction through tarsoconjunctival grafts and occurred in 9 of 44 patients over a 10-year period. ${ }^{7}$

\section{Trichiasis}

Malpositioned eyelashes, or trichiasis, is a complication that may arise from eyelid margin defects. ${ }^{7}$ Cutaneous malignancies that arise in close proximity to the lash line may predispose to this complication if repair is not carefully undertaken following excision. Trichiatic lashes that touch the ocular surface can lead to keratopathy, epithelial defects, and corneal ulceration that can threaten a patient's vision. Lid margin defects that are left to heal by secondary intention have been reported to result in trichiasis, although outcomes are typically acceptable ${ }^{15,16}$ Trichiasis may be isolated or associated with cicatricial entropion, and treatment is different depending on the context (see later in the article).

\section{Lid Margin Mucosal Overgrowth}

During the reconstructive healing, some patients experience conjunctival growth over the eyelid margin, resulting in an anterior mucocutaneous junction. This leads to the appearance of a red, inflamed eyelid margin. Patients with underlying blepharitis, Meibomian gland dysfunction, and ocular rosacea may be at a higher risk of this complication due to underlying lid margin inflammatory changes. ${ }^{17}$ Conjunctival overgrowth can be treated by gently cauterizing the conjunctiva back to the posterior lamella, leaving a raw skin edge, and allowing the mucocutaneous junction to reform.

\section{Lacrimal System Injury}

Periocular cutaneous surgery near the medial aspect of the lower eyelids potentially threatens the integrity of the lacrimal system. The lower eyelid punctum may require excision in cases of periocular malignancy with prepunctal and pericanalicular involvement. Surgery in the adjacent eyelid tissues may lead to punctal stenosis or punctal ectropion from inflammatory and cicatricial changes. Anatomically, the lower canaliculus runs inferior to the lower lid margin and typically forms a common canaliculus upon meeting the upper canaliculus just medial to the medial canthus. The tears flow through this system and empty into the lacrimal sac and nasolacrimal duct inferiorly. Tear drainage depends on a series of muscle contractions that result in a negative pressure system and tear suction into the sac. Injury to this region can damage the muscles and affect the functionality of this negative-pressure system. Although the canaliculi are most commonly affected in cases of malignancies overlying the lacrimal drainage system, excision and reconstruction of more aggressive vertically invasive cutaneous malignancies can potentially threaten the integrity of the more distal structures of the lacrimal drainage pathway. Postoperative cicatricial changes may also result in acquired nasolacrimal duct obstruction. Patients will present with complaints of tearing and findings of an increased tear meniscus and epiphora (overflow tearing) on ophthalmic examination. ${ }^{18}$

\section{Upper Eyelid Complications}

A key feature of the upper eyelid is that it is highly mobile, carrying the primary responsibility of covering the ocular surface through blinking and eyelid closure. It contains two vascular arcades along both edges of a wide tarsal plate: margin and peripheral arcade. The role of the peripheral arcade is to provide oxygen-carrying vascular supply to the otherwise avascular cornea during extended periods of eyelid closure, for example, sleep. Upper eyelid injuries 
and surgeries that lead to reduced mobility or compromise the vascular arcades can lead to ocular surface dysfunction, corneal disease, and vision loss. Furthermore, the upper eyelid is an extremely important element of facial expression, and terms such as "surprised look," "deer in the headlights," and "Betty Davis eyes" reflect the importance of the upper eyelid in facial expression and the conveying of emotions.

\section{Eyelid Retraction}

Retraction of the upper eyelids can occur from cicatricial changes and tissue loss following periocular surgery for cutaneous malignancies of the upper eyelid and brows. This mechanism is similar to lower eyelid retraction, as previously discussed, except that gravity is not an important factor, which is why upper eyelid retraction is much more common than upper eyelid ectropion, whereas lower eyelid retraction commonly accompanies lower eyelid ectropion. Upper eyelid retraction presents with superior scleral show, with the eyelid positioned above the superior corneal limbus. Eyelid retraction can be caused by loss of anterior lamellar (i.e., skin), as well as middle lamellar injury and scarring. The ideal amount of upper eyelid skin between the lash line and the eyelid-brow junction is at least $20 \mathrm{~mm}$ centrally.

\section{Poor Blink}

Damage to the upper eyelid retractors (levator and Mueller's muscles) and protractors (orbicular oculi) during excision of periocular cutaneous malignancies or subsequent reconstruction can damage the ability to blink. Iatrogenic facial nerve damage in the periocular region may also compromise blinking. Poor blink predisposes the ocular surface to exposure and may result in dry eye syndrome or keratopathy. Since the facial nerve innervates the orbicular oculi muscle from lateral to medial, injury to the lateral canthus can lead to partial orbicularis paresis and reduced blink-at least until the nerve regenerates, which can take months.

\section{Ptosis}

Surgical injury to the levator and/or Mueller's muscles can lead to myogenic eyelid ptosis. Cutaneous malignancies that involve the upper eyelid may predispose to injury of these structures during the excision process. Muscle damage may be inevitable if the malignancy extends deeply or is infiltrative in nature. However, with proper reconstruction of the eyelid, with attention to lamellar repair, ptosis often improves with time as the eyelid layers heal and muscle function returns. Measuring the eyelid excursion pre- and postoperatively, that is, assessing eyelid margin movement from downgaze to upgaze, can provide a quantitative measure that can be followed.

\section{Notching}

As in the lower lids, notching of the upper eyelid margin can occur following excision and reconstruction of periocular malignancies in cases where lid margin incisions are made. Please refer to the previous section for additional information. Because the upper eyelid is so much more mobile than the lower, central notching can lead to significant keratopathy as the notch moves across the corneal surface with each blink.

\section{Trichiasis}

The upper lid contains more cilia than the lower lid. By sheer volume, trichiatic lashes may be more frequently seen following surgery on the upper eyelid margin. As for notching, because the upper eyelid is so much more mobile than the lower, centrally located trichiatic lashes can lead to significant keratopathy as the lashes move across the corneal surface with each blink.

\section{Lateral Canthal Dystopia}

As in lower eyelid surgery for periocular cutaneous neoplasm, surgery on the upper eyelids may result in displacement of the normal lateral canthal anatomy. Please refer to the previous section for additional information.

\section{Webbing}

Medial canthal defects require repair after Mohs or direct excision of cutaneous malignancies. ${ }^{19}$ Subsequent reconstruction may result in medial webbing if the reconstruction creates vertical tension following wound repair. Excess anterior lamellar shortening may also cause webbing. Lateral canthal webbing may also occur but is much less common. The key to avoiding webbing is to replace vertical tension with horizontal tension.

\section{Lid Margin Mucosal Overgrowth}

As in lower eyelid surgery for periocular cutaneous neoplasm, surgery on the upper eyelids may result in mucosal overgrowth and hyperemia of the upper eyelid margin. Please refer to the previous section for additional information.

\section{Lacrimal System Injury}

Surgery on the medial upper eyelid may damage the upper canaliculus that runs superior to the eyelid margin or may injure the punctum. Damage may occur from excision of cutaneous malignancies that present in this region. Patients with lacrimal system damage involving the upper canaliculus or punctum may present with tearing, though they may be asymptomatic. The latter is particularly true in the case of elderly patients with reduced tear production ${ }^{20}$ and in patients who have sufficient lower canalicular function to drain basal tear secretion.

\section{Treatment}

\section{Avoiding Complications}

Periocular complications from the surgical management of cutaneous malignancies are best managed by preventing them in the first place with excellent surgical technique and preoperative planning.

To prevent eyelid positional abnormalities such as retraction and webbing, periocular tissue tension must be correctly distributed. Specifically, as much as possible, vertical tension must be converted into horizontal tension through proper design and the use of flaps and grafts., 21-25 Underlying 
eyelid laxity must be assessed as part of the preoperative evaluation and addressed preemptively during surgery. When needed, the lateral and medial canthal tendons should be tightened and tension reduced through the use of periosteal flaps or titanium microplate fixation as necessary. ${ }^{4}$

The need to avoid vertical tension distinguishes reconstruction of the periocular region from traditional teaching that emphasizes the minimization of horizontal tension by the formation of skin incisions along natural skin tension lines (Langer's lines). ${ }^{26}$

If necessary, any skin grafts used for periocular reconstruction must be oversized. Use of "pie crusting" incisions and bolsters can avoid the accumulation of blood underneath the graft, which could lead to graft failure and cicatricial changes. Additionally, to minimize scarring and inflammatory changes among the eyelid layers, septal injury should be avoided during the surgical process, if possible. We should be cognizant that the medial canthal area shows a higher propensity for graft hypertrophy, which may be prevented by placing grafts of similar color, texture, and histology that could be accomplished by combing several grafts of similar characteristics in that area. ${ }^{27}$ Medial canthal reconstruction is particularly amenable to nasal flaps including bilobed pedicle flaps, which avoid vertical tension (-Figs. 2 and $\mathbf{3}$ ). Details of the proper use of grafts and flaps in the periocular region are beyond the scope of this article, but readers are encouraged to review the outstanding descriptions provided in books by Shan Baker, David Tse, and Gerald Harris. ${ }^{28}$

Importantly, the various facial anatomical and aesthetic units should be respected and matched during the reconstructive process following excision of a cutaneous periocular malignancy. This is a particularly relevant consideration in the lateral and medial canthal regions where multiple aesthetic units (upper lid, lower lid, and canthus) overlap with unique skin thicknesses, texture, color, and contour. ${ }^{25,27}$ To avoid scarring or contracture in cases in which a periocular tumor transcends multiple aesthetic units, multiple skin grafts or flaps need to be used for each individual unit. ${ }^{29-31}$ For example, even if the upper eyelid defect is small, compared with a large nasal sidewall defect, each must be independently reconstructed: the medial upper eyelid defect with an eyelid musculocutaneous flap and the nasal sidewall with a nasal flap or skin graft. For optimal results, one should avoid placing a graft or using a flap across aesthetic units.

One reconstructive technique in particular to be mindful of is the Hughes procedure, in which a tarsoconjunctival graft is used to repair the posterior lamella of large full-thickness lower eyelid defects (-Fig. 4). The tarsoconjunctival flap provides a vascular bed onto which a skin graft can be placed. This is a twostage procedure, with the second stage to divide the flap and repair the eyelid margin. The tarsus should be incised such that at least $4 \mathrm{~mm}$ of the tarsal plate would be left for upper eyelid support. Mueller's muscle may be dissected off of the conjunctiva during flap construction, but this will lead to reduced vascular supply and increased risk of skin graft failure. Hence, in smokers and vasculopaths, Mueller's muscle should be advanced along with the tarsus and conjunctiva and recessed
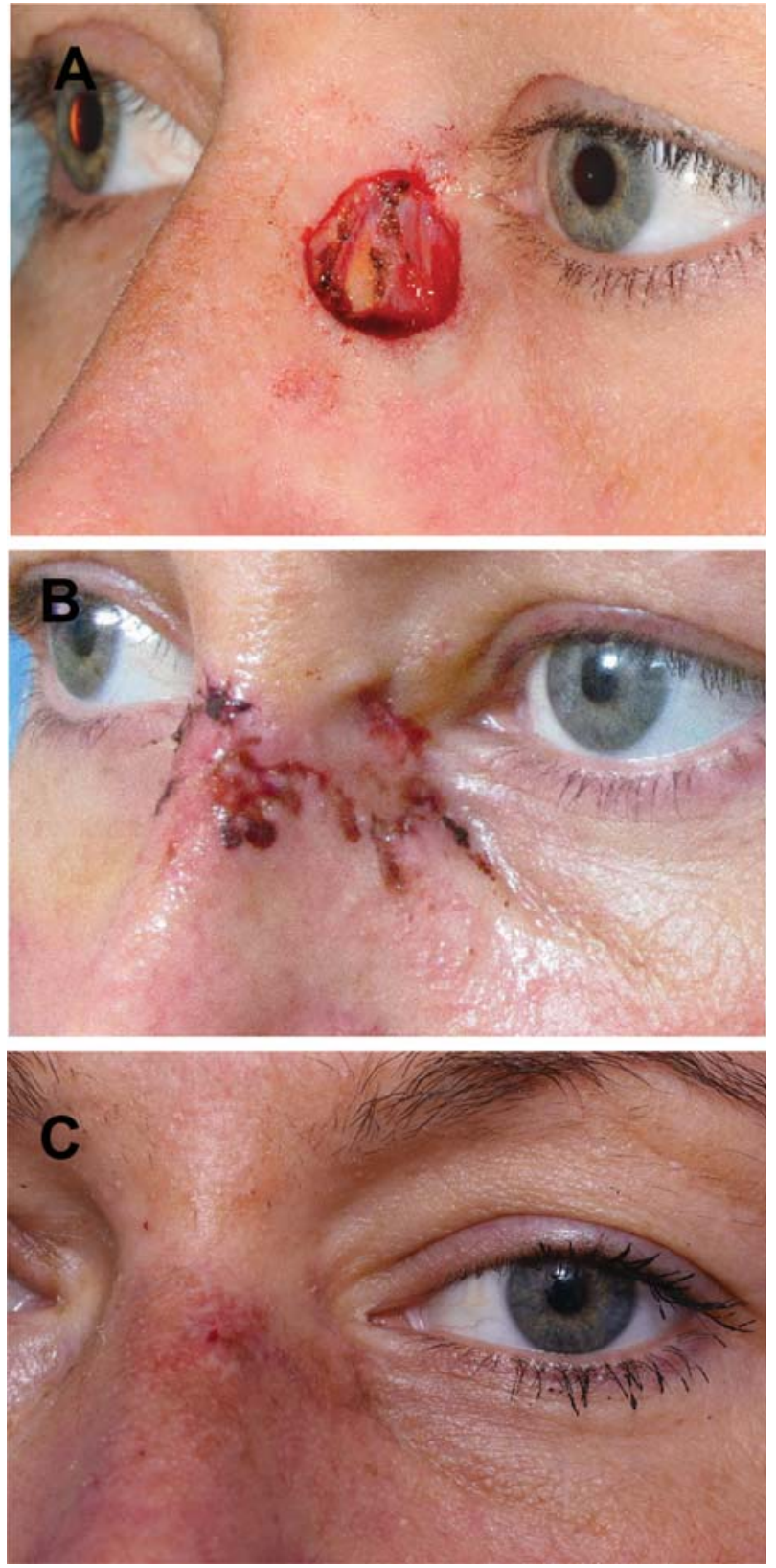

Fig. 2 Medial canthal tension redistribution with a bilobed flap. A young woman presented with basal cell carcinoma of the left medial canthus. Mohs micrographic surgery left her with a large defect $(A)$. A bilobed pedicle flap from the nasal dorsum (B) provided an adequate reconstruction with all tension directed medially, avoiding retraction or disfiguring scarring, as noted at her 3-month follow-up (C). With time, the erythema will subside and the scar will become imperceptible.

only during the second stage. In any case, Mueller's muscle should not be sacrificed, because Mueller's muscle resection will lead to eyelid retraction. ${ }^{32-34}$ However, when repairing a lower eyelid defect in which the upper eyelid is already significantly ptotic, intentional excision of Mueller's muscle can correct the upper eyelid ptosis-something that the lead author has used on occasion to improve overall eyelid functionality.

A very common occurrence following the Hughes procedure is an anterior displacement of the mucocutaneous junction, leading to the appearance of a red eyelid margin. This can be avoided by cauterizing the edge of the 

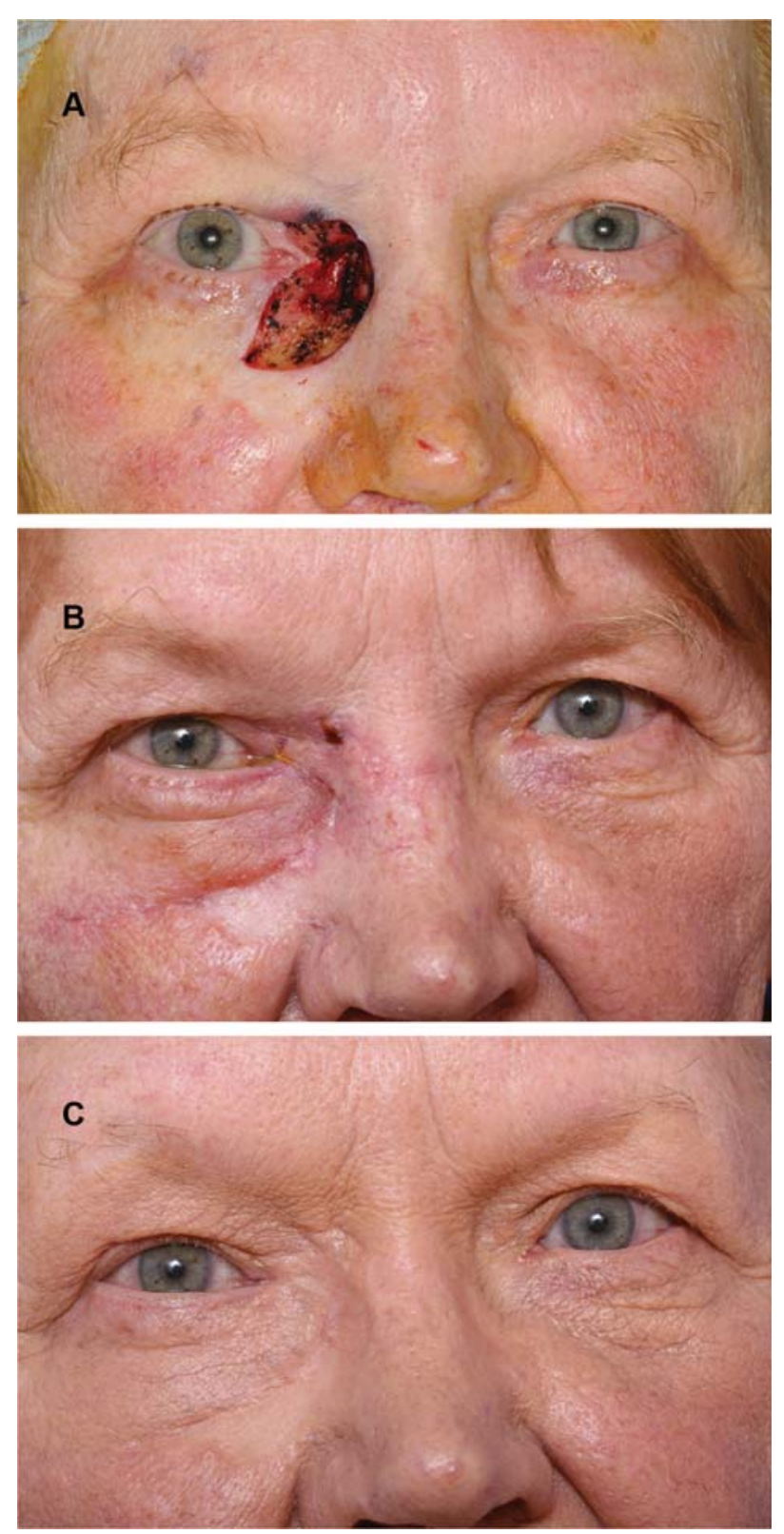

Fig. 3 Aesthetic units and redistribution of tension. The patient presented with a large recurrent right medial canthal basal cell carcinoma (no history of Mohs surgery). Following Mohs micrographic surgery, she had a large defect that crossed multiple aesthetic units, including the upper and lower eyelids, nasal sidewall, and cheek (A). Each aesthetic unit was repaired independently: a small myocutaneous advancement flap was used in the upper eyelid, a cheek flap with redistribution of tension was used in the lower eyelid and cheek, and a bilobed pedicle flap was used in the nasal sidewall (B). Sixmonth follow-up (C) revealed an adequate functional and aesthetic result. This patient was lucky that the cancer did not invade deeper into her orbit. In the opinion of the authors, for cutaneous carcinomas that involve the medial canthus, Mohs micrographic surgery should be the standard of care.

reconstructed eyelid at the time of the second stage to recess the conjunctiva posteriorly and the skin anteriorly and allowing the mucocutaneous junction to reform spontaneously. If the skin overgrows, it will lead to lid margin entropion and ocular surface irritation. More commonly, if the conjunctiva overgrows, it will lead to lid margin ectropion and redness. In either case, repeating the cauterizing process and adjusting the relative level of the skin and conjunctival edges will allow the margin to reform more correctly.

When reconstructing large upper lid defects, the Cutler Beard bridge flap serves as a viable solution; however, to minimize chances of lower lid ischemia, care should be taken to design the flap below the marginal arcade. ${ }^{34}$ Another option for smaller defects that still affect much of the upper eyelid margin is the "reverse Hughes," that is, the Leone procedure, for repairing full-thickness upper eyelid defects. ${ }^{35,36}$

Preoperatively, use of photographs is helpful in surgical planning. These photographs can be posted in the operating room at the time of surgery to help the surgeon remain aware of the patient's unique anatomical considerations and tailor the surgical plan to minimize eyelid complications. Intraoperatively, a good general guiding principle is that if the surgical result does not look good on the table, it certainly will not look good postoperatively.

Depending on the extent of defect, multiple unique techniques and approaches have been described to reduce the risk of postoperative eyelid complications from the initial surgical management of periocular cutaneous malignancy $^{22,23,37,38}$ (-Fig. 5).

\section{Managing Complications}

When postoperative complications do arise that affect the position, function, and cosmesis of the eyelid, scarring is often the culprit. Poor initial reattachment of the eyelid's delicate tissue layers and excess vertical tension can cause cicatricial changes. Not only do patients often dislike their cosmetic result in cases of postoperative scarring, but they are also at a risk of sustaining damage to the cornea and globe itself from excess ocular exposure, trichiasis, and inadequate blink.

Repair of malpositioned eyelids requires a nuanced, sometimes staged, approach. First, adequate time should be given to healing, often at least 4 to 6 months unless there is active keratopathy that cannot be managed with lubrication. Postoperative injections of 5-fluorouracil can reduce scarring and improve tissue suppleness. ${ }^{39}$ If there is active ocular surface disease, then earlier surgery is indicated, and in these cases, often the closer the secondary surgery is to the primary surgery, the better.

Once the surgeon determines that reconstructive surgery is required, the goal should be complete release of the scar tissue and elimination of any vertical tension followed by reconstruction of the resulting defect "from scratch." $8,40,41$ Any laxity should be addressed at this time, and any grafts or flaps should be significantly oversized (-Fig. 6). Other options for scar minimization are pressure garments, steroid injections, massages, or silicone gel sheets. ${ }^{42}$ Any residual eyelid laxity following initial repair may predispose to lower eyelid retraction, exposure keratopathy, and conjunctival irritation. This is often a greater concern in older patients with aging skin and may be associated with eyelid margin instability, which can result in entropion or ectropion. Surgical reconstruction of any postoperative eyelid complication where laxity is present should be addressed and the 

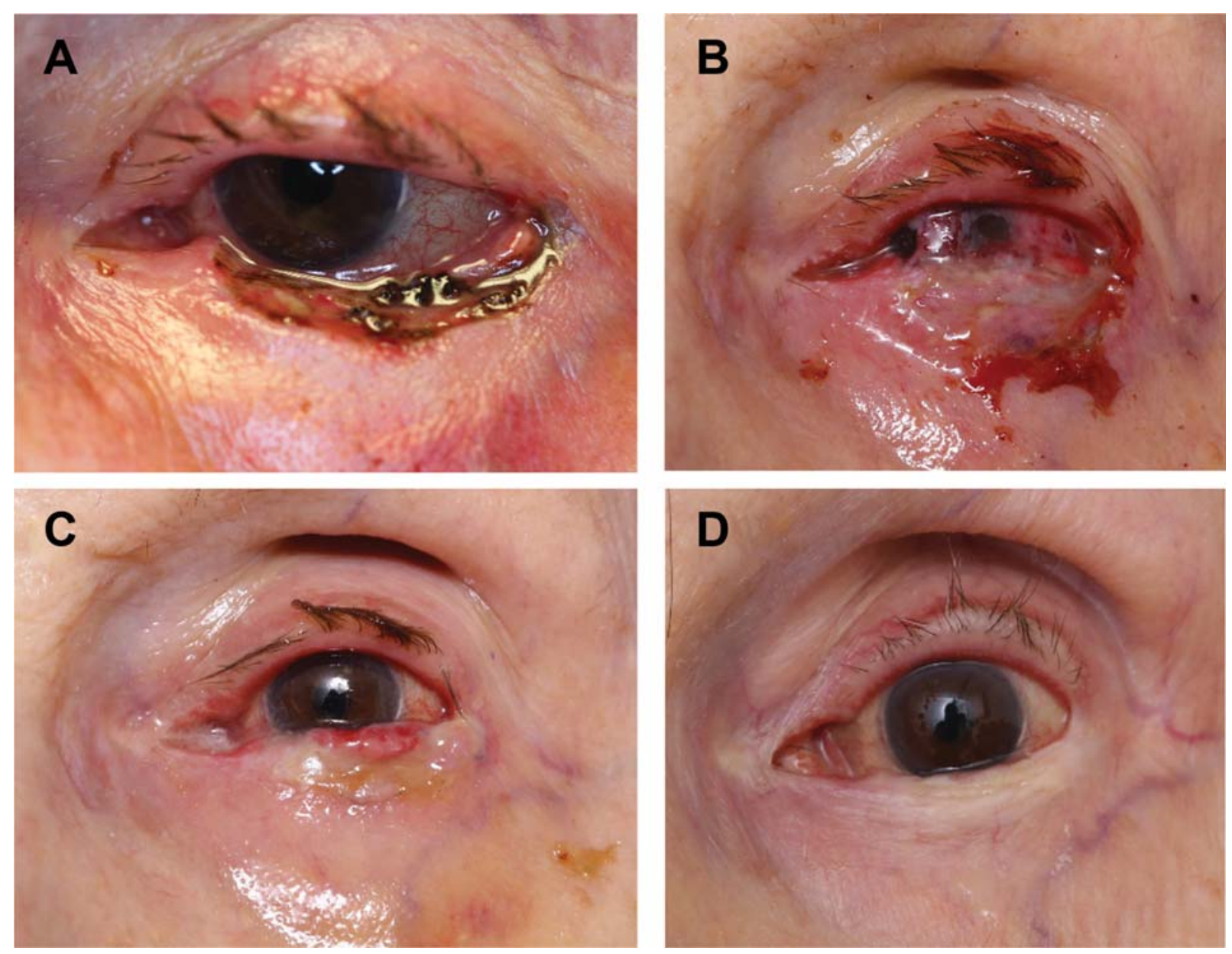

Fig. 4 Hughes tarsoconjunctival flap. The Hughes tarsoconjunctival flap with a skin graft is used to reconstruct an eyelid that has lost most of its margin structure. The presence of a small lateral eyelid margin stump can assist in anchoring the tarsal component of the flap. In its absence, a periosteal flap is required. The proximity of the punctum to the medial edge of the defect $(A)$ creates a challenge, and care must be taken in placement of the sutures (B). In patients who are not vasculopaths, Mueller's muscle can be dissected off of the conjunctiva to create a very thin conjunctival flap (B) that provides sufficient vascular supply to sustain a skin graft. During or following the second stage (C), mucosa that extends over the newly created eyelid margin can be treated and recessed with high-temperature cautery. The final result (D) is a functional eyelid that also reconstructs the appearance. Note that the skin graft, taken from the upper eyelid, is still slightly discolored compared with the rest of the eyelid (D).

aim should be to repair the lax eyelid. Lateral or medial canthal plication or canthopexy ${ }^{43}$ with medial tendon fixation posterior to the lacrimal sac, ${ }^{44}$ lateral tarsal strip procedure with lateral tendon fixation inside the orbital $\mathrm{rim}^{45}$, or horizontal eyelid-shortening through wedge excision or Bick's procedure ${ }^{46}$ with or without addressing the vertical vector cheek lift $^{47-49}$ ( - Fig. 7) can be a viable option depending on the severity of laxity and the needs of the individual patient. ${ }^{50}$ Mild laxity may respond well to simple canthopexy, whereas more extensive laxity may require eyelid shortening procedures. ${ }^{51}$

As mentioned earlier in our discussion of complication prevention during primary surgery, any skin grafts that are used in repeat surgery to address anterior lamellar defects should also be oversized, with "pie crusting" incisions and bolsters. These approaches mitigate the accumulation of blood under the graft and reduce the risk of graft contraction.

Spacer grafts may be used to manage lower eyelid retraction, which is often caused by lower lid retractor tethering and septal scarring resulting from cicatricial postoperative changes. Various spacer graft materials, including hard palate mucosa, ${ }^{52}$ acellular dermal matrix, ${ }^{53,54}$ autogenous tarsus, ${ }^{55}$ and ear cartilage, ${ }^{56}$ may be interpostitioned following recession of the lower eyelid retractors to elevate the height of the lower lid. Lysis of the midlamellar scarring can aid the lower lid retraction in cases of postoperative midlamella shrinking. ${ }^{57} \mathrm{To}$ prevent or correct ectropion after surgery near the lower eyelid, a Frost suture can be placed to maintain upward tension on the lower eyelid. ${ }^{58}$ In contrast to lower lid retraction, upper eyelid retraction is usually managed without spacer grafting: with a skin graft for retraction caused by anterior lamellar shortening, and a "blepharotomy" technique without spacer grafting for retraction caused by posterior lamellar shortening. For posterior lamellar shortening, both anterior and posterior approaches exist for the blepharotomy technique. Both are effective, although the senior author (A.K.) of this study prefers the posterior approach, leaving the skin and orbicularis muscle unaltered. 

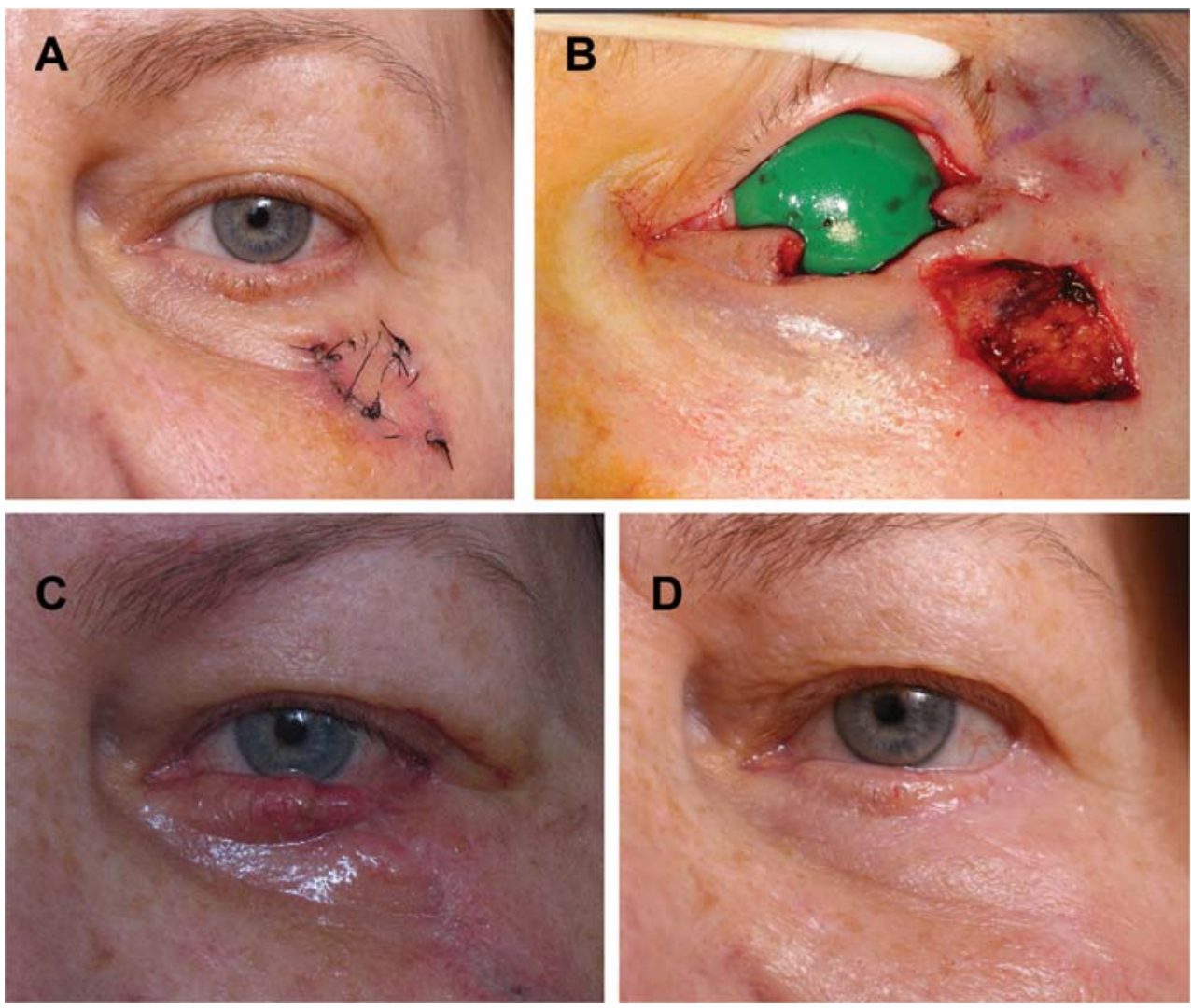

Fig. 5 Tension redistribution and Tenzel flap. The patient presented with melanoma in situ of the left cheek, as well as basal cell carcinoma of the left lower eyelid margin. Following staged two-blade margin control of the melanoma in situ (A), the melanoma and basal cell carcinoma were excited at the same time (B). The key to this reconstruction was distribution of tension. With an aggressive cheek lift, sufficient skin was developed to repair the cheek defect with a Zflap and to repair the eyelid defect with a "Tenzel" semicircular flap (C). The result (D) reveals a functional eyelid that is aesthetically adequate, with slight rounding of the lateral aspect of the lower eyelid.
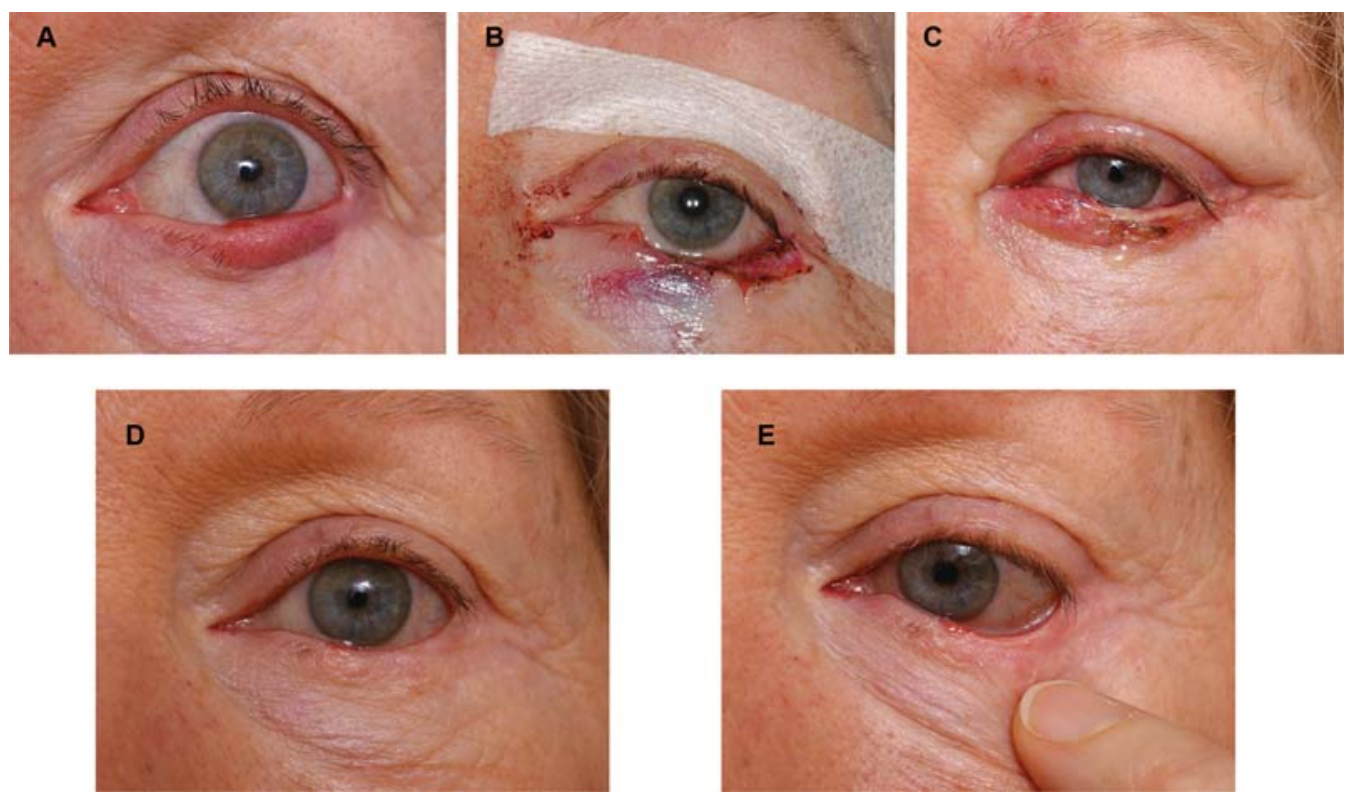

Fig. 6 Eyelid margin conjunctival overgrowth. The patient presented with a recurrent left lower eyelid basal cell carcinoma (A) that had been previously excised and reconstructed with a Hughes flap by a different surgeon. Hence, a Hughes flap could no longer be used, posing a major challenge. Mohs micrographic surgery resulted in a near-total loss of the lower eyelid margin as well as the lateral commissure (B). A "Tenzel" semicircular flap was used for the anterior lamella. A lateral orbital rim periosteal flap was used for the posterior lamella, which was angled to pull the eyelid superolaterally (C). A lateral cheek lift was performed, with anchoring to the periosteum, to distribute tension away from the eyelid-a critical step in the reconstructive process. The result (D,E) reveals a well-formed functional eyelid but with a small area of conjunctival overgrowth at the medial surgical margin (E). This was successfully treated with high-temperature cautery to recess the conjunctival overgrowth. 

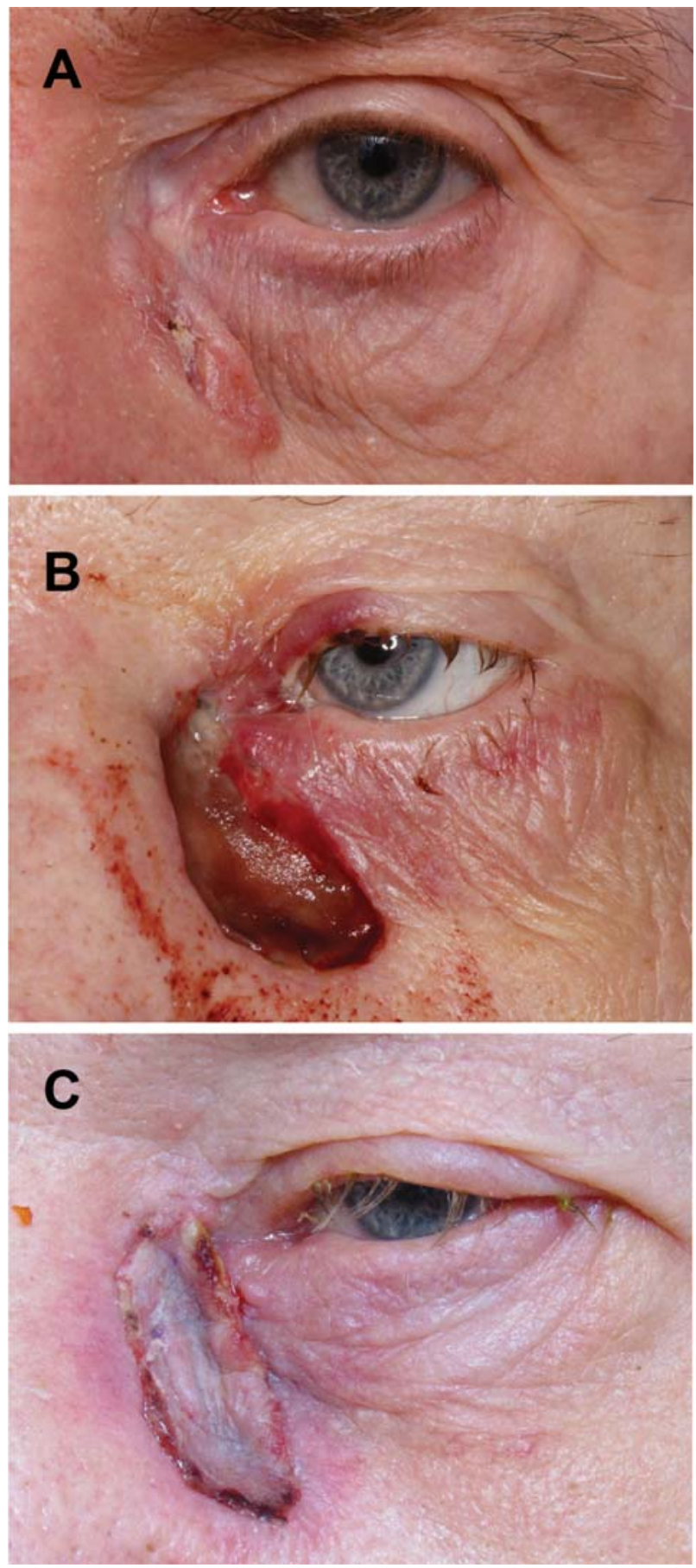

Fig. 7 Skin graft and tension redistribution. The patient presented with a recurrent left medial canthal basal cell carcinoma extending onto the cheek $(\mathrm{A})$. Mohs micrographic surgery resulted in a deep defect that crossed aesthetic units (B). To reduce the risk of medial eyelid retraction, a medial cheek lift was performed to redistribute tension followed by a full-thickness skin graft; 1-week postoperative result revealed excellent lower eyelid position (C).

As is the case for primary surgical intervention for periocular cutaneous malignancies, it is important to avoid vertical tension and respect aesthetic units during any repeat surgery that aims to correct complications from initial excision and/or repair.

\section{Conclusion}

The best way to treat an eyelid complication from surgery for periocular cutaneous malignancy is to avoid it in the first place. Skin closure must be meticulous, and surgery must be planned and performed thoughtfully to avoid vertical tension, respect aesthetic units, and address laxity.

\section{Conflict of Interest}

The authors have no relevant conflicts of interest to report.

\section{References}

1 Yelin JB, Clayton-Dyer BJ, Naderi A, Esmaeli B. Eyelid reconstruction: an oculoplastic surgeon's perspective. Plast Surg Nurs 2001; 21(04):192-195, 201

2 Miller PK, Roenigk RK, Brodland DG, Randle HW. Cutaneous micrographic surgery: Mohs procedure. Mayo Clin Proc 1992; 67(10):971-980

3 Moskowitz BK, Patel AD, Pearson JM. Aesthetic and functional management of eyelid and orbital reconstruction. Facial Plast Surg 2008;24(01):069-77

4 Hayano SM, Whipple KM, Korn BS, Kikkawa DO. Principles of periocular reconstruction following excision of cutaneous malignancy. J Skin Cancer 2012;2012:438502

5 Gosain AK, Yousif NJ, Madiedo G, Larson DL, Matloub HS, Sanger JR. Surgical anatomy of the SMAS: a reinvestigation. Plast Reconstr Surg 1993;92(07):1254-1263, discussion 1264-1265

6 Rubin P, Mykula R, Griffiths RW. Ectropion following excision of lower eyelid tumours and full thickness skin graft repair. Br J Plast Surg 2005;58(03):353-360

7 Hawes MJ, Jamell GA. Complications of tarsoconjunctival grafts. Ophthal Plast Reconstr Surg 1996;12(01):45-50

8 Chi M, Kim HJ, Vagefi R, Kersten RC. Modified tarsotomy for the treatment of severe cicatricial entropion. Eye (Lond) 2016;30(07): 992-997

9 Black EH, Nesi FA, Gladstone GJ, Levine MR. Smith and Nesi's Ophthalmic Plastic and Reconstructive Surgery. Black EH, Nesi FA, Gladstone GJ, Levine MR, eds. 3rd ed. New York, NY: Springer; 2012

10 Gossman MD. Lateral Canthal Complications in Aesthetic Eyelid Surgery: Prevention and Reconstruction. Available at: https:// eyelidsurgerylouisville.com/2017/04/04/lateral-canthal-complications-in-aesthetic-eyelid-surgery-prevention-and-reconstruction/. Accessed February 17, 2020

11 Patipa M. The evaluation and management of lower eyelid retraction following cosmetic surgery. Plast Reconstr Surg 2000;106(02):438-453, discussion 454-459

12 Goldberg RA, Lessner AM, Shorr N, Baylis HI. The transconjunctival approach to the orbital floor and orbital fat. A prospective study. Ophthal Plast Reconstr Surg 1990;6(04):241-246

13 Latkany RL, Lock B, Speaker M. Nocturnal lagophthalmos: an overview and classification. Ocul Surf 2006;4(01):44-53

14 Divine RD, Anderson RL. Techniques in eyelid wound closure. Ophthalmic Surg 1982;13(04):283-287

15 Lowry JC, Bartley GB, Garrity JA. The role of second-intention healing in periocular reconstruction. Ophthal Plast Reconstr Surg 1997;13(03):174-188

16 Trieu DN, Drosou A, White LE, Goldberg LH. Outcomes of second intention healing of the lower eyelid margin after Mohs micrographic surgery. Dermatol Surg 2019;45(07):884-889

17 Suzuki T. Meibomitis-related keratoconjunctivitis: implications and clinical significance of meibomian gland inflammation. Cornea 2012;31(Suppl 1):S41-S44 
18 Mills DM, Meyer DR. Acquired nasolacrimal duct obstruction. Otolaryngol Clin North Am 2006;39(05):979-999, vii

$19 \mathrm{Ng} \mathrm{SG}$, Inkster CF, Leatherbarrow B. The rhomboid flap in medial canthal reconstruction. Br J Ophthalmol 2001;85(05):556-559

20 Sharma A, Hindman HB. Aging: a predisposition to dry eyes. J Ophthalmol 2014;2014(03):781683

21 Saito A, Saito N, Furukawa H, et al. Reconstruction of periorbital defects following malignant tumour excision: a report of 50 cases. J Plast Reconstr Aesthet Surg 2012;65(05):665-670

22 Harris GJ, Perez N. Anchored flaps in post-Mohs reconstruction of the lower eyelid, cheek, and lateral canthus: avoiding eyelid distortion. Ophthal Plast Reconstr Surg 2003;19(01):5-13

23 Khan JA, Garden VS. Combined flap repair of moderate lower eyelid defects. Ophthal Plast Reconstr Surg 2002;18(03):202-204

24 Wójcicki P, Zachara M. Surgical treatment of eyelid tumors. J Craniofac Surg 2010;21(02):520-525

25 Bowman PH, Fosko SW, Hartstein ME. Periocular reconstruction. Semin Cutan Med Surg 2003;22(04):263-272

26 Langer K. On the anatomy and physiology of the skin: I. The cleavability of the cutis. Br J Plast Surg 1978;31(01):3-8

27 Leibovitch I, Huilgol SC, Hsuan JD, Selva D. Incidence of host site complications in periocular full thickness skin grafts. Br J Ophthalmol 2005;89(02):219-222

28 Baker Sed. Local Flaps in Facial Reconstruction. 3rd ed. Philadelphia, PA: Elsevier/Saunders; 2014

29 Harris GJ, Logani SC. Multiple aesthetic unit flaps for medial canthal reconstruction. Ophthal Plast Reconstr Surg 1998;14 (05):352-359

30 Maillard GF, Clavel PR. Aesthetic units in skin grafting of the face. Ann Plast Surg 1991;26(04):347-352

31 Gonzalez-Ulloa M. Restoration of the face covering by means of selected skin in regional aesthetic units. Br J Plast Surg 1956;9 (03):212-221

32 Rohrich RJ, Zbar RI. The evolution of the Hughes tarsoconjunctival flap for the lower eyelid reconstruction. Plast Reconstr Surg 1999; 104(02):518-22; quiz 23; discussion 24-6

33 Huggins A, Latting M, Marx D, Giacometti J. Ocular adnexal reconstruction for cutaneous periocular malignancies. Semin Plast Surg 2017;31(01):22-30

34 Gill P, Bidros R, Boutros S. Reconstruction of oncologic defects of the eyelid, lips and ear [review article]. Indian J Plast Surg 2007;40 (12):13

35 Leone CR Jr. Tarsal-conjunctival advancement flaps for upper eyelid reconstruction. Arch Ophthalmol 1983;101(06):945-948

36 Jordan DR, Anderson RL, Nowinski TS. Tarsoconjunctival flap for upper eyelid reconstruction. Arch Ophthalmol 1989;107(04):599-603

37 Kaufman AJ. Periorbital reconstruction with adjacent-tissue skin grafts. Dermatol Surg 2005;31(12):1704-1706

38 Mathijssen IM, van der Meulen JC. Guidelines for reconstruction of the eyelids and canthal regions. J Plast Reconstr Aesthet Surg 2010;63(09):1420-1433

39 Yoo DB, Azizzadeh B, Massry GG. Injectable 5-FU with or without added steroid in periorbital skin grafting: initial observations. Ophthal Plast Reconstr Surg 2015;31(02):122-126

40 Levin ML, Leone CR Jr. Bipedicle myocutaneous flap repair of cicatricial ectropion. Ophthal Plast Reconstr Surg 1990;6(02):119-121
41 Choi CJ, Bauza A, Yoon MK, Sobel RK, Freitag SK. Full-thickness skin graft as an independent or adjunctive technique for repair of cicatricial lower eyelid ectropion secondary to actinic skin changes. Ophthal Plast Reconstr Surg 2015;31(06):474-477

42 Tredget EE, Nedelec B, Scott PG, Ghahary A. Hypertrophic scars, keloids, and contractures. The cellular and molecular basis for therapy. Surg Clin North Am 1997;77(03):701-730

43 Ishida Y, Takahashi Y, Kakizaki H. Posterior layer advancement of lower eyelid retractors with transcanthal canthopexy for involutional lower eyelid entropion. Eye (Lond) 2016;30(11): 1469-1474

44 Sassoon EM, Codner MA. Eyelid reconstruction. Oper Tech Plast Reconstr Sur 1999;6(04):250-264

45 Anderson RL, Gordy DD. The tarsal strip procedure. Arch Ophthalmol 1979;97(11):2192-2196

46 Bick MW. Surgical management of orbital tarsal disparity. Arch Ophthalmol 1966;75(03):386-389

47 Salgarelli AC, Francomano M, Magnoni C, Bellini P. Cicatricial iatrogenic lower eyelid malposition in skin cancer surgery: results of a combined approach. J Craniomaxillofac Surg 2012;40(07): 579-583

48 Kahana A, Lucarelli MJ. Adjunctive transcanthotomy lateral suborbicularis fat lift and orbitomalar ligament resuspension in lower eyelid ectropion repair. Ophthal Plast Reconstr Surg 2009;25(01):1-6

49 Marshak H, Morrow DM, Dresner SC. Small incision preperiosteal midface lift for correction of lower eyelid retraction. Ophthal Plast Reconstr Surg 2010;26(03):176-181

50 Pascali M, Corsi A, Brinci L, Corsi I, Cervelli V. The tarsal belt procedure for the correction of ectropion: description and outcome in 42 cases. Br J Ophthalmol 2014;98(12):1691-1696

51 Mupas-Uy JT, Takahashi Y, Nakano T, et al. Interlower and postlower eyelid retractor fat pads: a cadaveric microscopic study. $\mathrm{Br} \mathrm{J}$ Ophthalmol 2018;102(03):404-406

52 Kersten RC, Kulwin DR, Levartovsky S, Tiradellis H, Tse DT. Management of lower-lid retraction with hard-palate mucosa grafting. Arch Ophthalmol 1990;108(09):1339-1343

53 McCord C, Nahai FR, Codner MA, Nahai F, Hester TR. Use of porcine acellular dermal matrix (Enduragen) grafts in eyelids: a review of 69 patients and 129 eyelids. Plast Reconstr Surg 2008;122(04): 1206-1213

54 Rubin PA, Fay AM, Remulla HD, Maus M. Ophthalmic plastic applications of acellular dermal allografts. Ophthalmology 1999;106(11):2091-2097

55 Guthoff R, Guthoff T, Mueller-Hermelink HK, Sold-Darseff J, Geissinger E. Perivascular epithelioid cell tumor of the orbit. Arch Ophthalmol 2008;126(07):1009-1011

56 Beigi B, Khandwala M, Degoumois A, Ogbuehi KC, Gupta D. Lower eyelid excursion: a functional and cosmetically relevant parameter in the treatment of lower eyelid retraction. J Plast Reconstr Aesthet Surg 2019;72(02):310-316

57 Long JA, Gutta R. Orbital, periorbital, and ocular reconstruction. Oral Maxillofac Surg Clin North Am 2013;25(02):151-166

58 Connolly KL, Albertini JG, Miller CJ, Ozog DM. The suspension (Frost) suture: experience and applications. Dermatol Surg 2015; 41(03):406-410 